\title{
Quark sea asymmetries of the octet baryons
}

\author{
Neetika Sharma and Harleen Dahiya \\ Department of Physics, \\ Dr. B.R. Ambedkar National Institute of Technology, \\ Jalandhar, 144011, India
}

The effects of "quark sea" in determining the flavor structure of the octet baryons have been investigated in the chiral constituent quark model $(\chi \mathrm{CQM})$. The $\chi \mathrm{CQM}$ is able to qualitatively generate the requisite amount of quark sea and is also known to provide a satisfactory explanation of the proton spin and related issues in the nonperturbative regime. The Bjorken scaling variable $x$ has been included phenomenologically in the sea quark distribution functions to understand its implications on the quark sea asymmetries like $\bar{d}(x)-\bar{u}(x), \bar{d}(x) / \bar{u}(x)$ and Gottfried integral for the octet baryons. The results strengthen the importance of quark sea at lower values of $x$. 
After the first direct evidence for the point-like constituents in the nucleon 1, identified as the valence quarks with spin-1/2 in the naive constituent quark model (NQM) [2 4, a lot of experiments have been conducted to probe the structure of the proton in the deep inelastic scattering (DIS) experiments. Surprisingly, the DIS results in the early 80's [5] indicated that the valence quarks of the proton carry only about $30 \%$ of its spin and is referred to as the "proton spin crisis" in the NQM. These results provided the first evidence for the proton being composed of three valence quarks surrounded by an indistinct sea of quark-antiquark pairs (henceforth referred to as the "quark sea"). In the present day, the study of the composition of hadrons can be said to be primarily the study of the quark sea and gluons and is considered as one of the active areas in hadronic physics.

The conventional expectation that the quark sea perhaps can be obtained through the perturbative production of the quark-antiquark pairs by gluons produces nearly equal numbers of $\bar{u}$ and $\bar{d}$. Until early 90 's a symmetric sea w.r.t. $\bar{u}$ and $\bar{d}$ was assumed, however, the famous New Muon Collaboration in 1991 [6] established the quark sea asymmetry of the unpolarized quarks in the case of nucleon by measuring $\bar{d}-\bar{u}$ giving first clear evidence for the nonperturbative origin of the quark sea. This was later confirmed by the Drell-Yan experiments [7] which measured a large quark sea asymmetry ratio $\bar{d} / \bar{u}$ reminding us that the study of the quark sea is intrinsically a nonperturbative phenomena and it is still a big challenge to perform these calculations from the first principles of QCD.

One approach to account for the observed quark sea asymmetry is the pion cloud mechanism [8] where the quark sea is believed to originate from process such as virtual pion production. It is suggested that in the deep inelastic lepton-nucleon scattering, the lepton probe also scatters off the pion cloud surrounding the target proton. The $\pi^{+}(\bar{d} u)$ cloud, dominant in the process $p \rightarrow \pi^{+} n$, leads to an excess of $\bar{d}$ sea. However, this effect should be significantly reduced by the emissions such as $p \rightarrow \Delta^{++}+\pi^{-}$with $\pi^{-}(\bar{u} d)$ cloud. Therefore, the pion cloud idea is not able to explain the significant $\bar{d}>\bar{u}$ asymmetry. This approach can be improved upon by adopting a mechanism which operates in the interior of the hadron.

The chiral constituent quark model $(\chi \mathrm{CQM})$ 9 can yield an adequate description of the quark sea generation through the chiral fluctuations. The basic idea is based on the possibility that chiral symmetry breaking takes place at a distance scale much smaller than the confinement scale. In this region, the effective degrees of freedom are the valence quarks and the internal Goldstone bosons (GBs) which are coupled to the valence quarks [10 12] allowing a simple and intuitive method to investigate the principle features of the hadron structure. In the case of spin dependent quantities, the $\chi \mathrm{CQM}$ is not only successful in giving a satisfactory explanation of "proton spin crisis" [10, 13] but is also able to account for the baryon magnetic moments [14] and hyperon $\beta$-decay parameters [11, 15, 16]. However, in the case of quark distribution functions, the latest developments by the NuSea (E866) [17] and HERMES [18] to determine the variation of the sea-antiquark ratio $\bar{d}(x) / \bar{u}(x)$ and the difference $\bar{d}(x)-\bar{u}(x)$ with Bjorken scaling variable $x$ have renewed considerable interest in the quark sea asymmetries.

Recently, there has been substantial theoretical progress to take into account the effects of quark sea in determining the flavor structure of the baryons and the question of sea asymmetry has been investigated by several authors using various phenomenological models. Calculations have been carried out in the meson cloud models [19], chiral quarksoliton model [20, effective chiral quark model [21], statistical models 22], bag model [23, model for parton densities 24], radiative parton model [25] etc.. However, the inclusion of $x$-dependence has not yet been successfully included in the quark distribution functions. Therefore, pending further experiments, it would be interesting to examine the flavor structure of the octet baryons at low energy, thereby giving vital clues to the nonperturbative effects of QCD. The study of $x$-dependence in the quark distribution functions becomes particularly interesting for the $\chi \mathrm{CQM}$ where the effects of quark sea and valence quarks can separately be calculated.

The purpose of the present communication is to determine the sea quark distribution functions and their asymmetries in the octet baryons by phenomenologically incorporating $x$-dependence in the $\chi \mathrm{CQM}$. The extent of contributions coming from the different sea quarks for the octet baryons can also be compared. To understand the relation of the Bjorken scaling variable and quark sea, it would be significant to study its implications in the region $x<0.3$ which is a relatively clean region to test the quark sea structure as well as to estimate their structure functions and related quantities 26 .

The key to understand the "proton spin crisis", in the $\chi \mathrm{CQM}$ formalism [11], is the fluctuation process

$$
q^{ \pm} \rightarrow \mathrm{GB}+q^{\prime} \mp \rightarrow\left(q \bar{q}^{\prime}\right)+q^{\prime} \mp,
$$

$q \bar{q}^{\prime}+q^{\prime}$ constitute the "quark sea" [11-13, 15]. The effective Lagrangian describing interaction between quarks and a nonet of GBs, can be expressed as

$$
\mathcal{L}=g_{8} \overline{\mathbf{q}}\left(\Phi+\zeta \frac{\eta^{\prime}}{\sqrt{3}} I\right) \mathbf{q}=g_{8} \overline{\mathbf{q}}\left(\Phi^{\prime}\right) \mathbf{q}
$$

where $\zeta=g_{1} / g_{8}, g_{1}$ and $g_{8}$ are the coupling constants for the singlet and octet GBs, respectively, $I$ is the $3 \times 3$ identity matrix. In terms of the $\mathrm{SU}(3)$ and axial $\mathrm{U}(1)$ symmetry breaking parameters, introduced by considering $M_{s}>M_{u, d}$, 
$M_{K, \eta}>M_{\pi}$ and $M_{\eta^{\prime}}>M_{K, \eta}[11,12$, 15, the GB field can be expressed as

$$
\Phi^{\prime}=\left(\begin{array}{ccc}
\frac{\pi^{0}}{\sqrt{2}}+\beta \frac{\eta}{\sqrt{6}}+\zeta \frac{\eta^{\prime}}{\sqrt{3}} & \pi^{+} & \alpha K^{+} \\
\pi^{-} & -\frac{\pi^{0}}{\sqrt{2}}+\beta \frac{\eta}{\sqrt{6}}+\zeta \frac{\eta^{\prime}}{\sqrt{3}} & \alpha K^{0} \\
\alpha K^{-} & \alpha \bar{K}^{0} & -\beta \frac{2 \eta}{\sqrt{6}}+\zeta \frac{\eta^{\prime}}{\sqrt{3}}
\end{array}\right) \text { and } q=\left(\begin{array}{l}
u \\
d \\
s
\end{array}\right) .
$$

The parameter $a\left(=\left|g_{8}\right|^{2}\right)$ denotes the probability of chiral fluctuation $u(d) \rightarrow d(u)+\pi^{+(-)}$, whereas $\alpha^{2} a, \beta^{2} a$ and $\zeta^{2} a$ respectively denote the probabilities of fluctuations $u(d) \rightarrow s+K^{-(0)}, u(d, s) \rightarrow u(d, s)+\eta$, and $u(d, s) \rightarrow u(d, s)+\eta^{\prime}$.

For the sake of simplification, the GB field can also be expressed in terms of the quark contents of the GBs and is expressed as

$$
\Phi^{\prime}=\left(\begin{array}{ccc}
\phi_{u u} u \bar{u}+\phi_{u d} d \bar{d}+\phi_{u s} s \bar{s} & \varphi_{u d} u \bar{d} & \varphi_{u s} u \bar{s} \\
\varphi_{d u} d \bar{u} & \phi_{d u} u \bar{u}+\phi_{d d} d \bar{d}+\phi_{d s} s \bar{s} & \varphi_{d s} d \bar{s} \\
\varphi_{s u} s \bar{u} & \phi_{s d} s \bar{d} & \phi_{s u} u \bar{u}+\phi_{s d} d \bar{d}+\phi_{s s} s \bar{s}
\end{array}\right)
$$

where

$$
\begin{aligned}
& \phi_{u u}=\phi_{d d}=\frac{1}{2}+\frac{\beta}{6}+\frac{\zeta}{3}, \quad \phi_{s s}=\frac{2 \beta}{3}+\frac{\zeta}{3}, \quad \phi_{u s}=\phi_{d s}=\phi_{s u}=\phi_{s d}=-\frac{\beta}{3}+\frac{\zeta}{3}, \\
& \phi_{d u}=\phi_{u d}=-\frac{1}{2}+\frac{\beta}{6}+\frac{\zeta}{3}, \quad \varphi_{u d}=\varphi_{d u}=1, \quad \varphi_{u s}=\varphi_{d s}=\varphi_{s u}=\varphi_{s d}=\alpha .
\end{aligned}
$$

The quark sea content of the baryon can be calculated in $\chi$ CQM by substituting for every constituent quark $q \rightarrow$ $\sum P_{q} q+|\psi(q)|^{2}$, where $\sum P_{q}$ is the transition probability of the emission of a GB from any of the $q$ quark and $|\psi(q)|^{2}$ is the transition probability of the $q$ quark. The flavor structure for the baryon of the type $B(x x y)$ is expressed as $2 P_{x} x+P_{y} y+2|\psi(x)|^{2}+|\psi(y)|^{2}$ and for the type $B(x y z)$ it is expressed as $P_{x} x+P_{y} y+P_{z} z+|\psi(x)|^{2}+|\psi(y)|^{2}+|\psi(z)|^{2}$, where $x, y, z=u, d, s$. The sea quark distribution function for the octet baryons $p, \Sigma^{+}, \Sigma^{0}$, and $\Xi^{0}$ have been presented in Table [

\begin{tabular}{|c|c|c|c|}
\hline Baryon & $\bar{u}$ & $\bar{d}$ & $\bar{s}$ \\
\hline$p(u u d)$ & $a\left(2 \phi_{u u}^{2}+\phi_{d u}^{2}+\varphi_{d u}^{2}\right)$ & $a\left(2 \phi_{u d}^{2}+2 \varphi_{u d}^{2}+\phi_{d d}^{2}\right)$ & $a\left(2 \phi_{u s}^{2}+2 \varphi_{u s}^{2}+\phi_{d s}^{2}+\varphi_{u s}^{2}\right)$ \\
$\Sigma^{+}(u u s)$ & $a\left(2 \phi_{u u}^{2}+\phi_{s u}^{2}+\varphi_{s u}^{2}\right)$ & $a\left(2 \phi_{u d}^{2}+2 \varphi_{u d}^{2}+\phi_{s d}^{2}+\varphi_{s d}^{2}\right)$ & $a\left(2 \phi_{u s}^{2}+2 \varphi_{u s}^{2}+\phi_{s s}^{2}\right)$ \\
$\Sigma^{0}(u d s)$ & $a\left(\phi_{u u}^{2}+\phi_{d u}^{2}+\phi_{s u}^{2}+\varphi_{d u}^{2}+\varphi_{s u}^{2}\right)$ & $a\left(\phi_{u d}^{2}+\phi_{d d}^{2}+\phi_{s d}^{2}+\varphi_{u d}^{2}+\varphi_{s d}^{2}\right)$ & $a\left(\phi_{u s}^{2}+\phi_{d s}^{2}+\phi_{s s}^{2}+\varphi_{u s}^{2}+\varphi_{d s}^{2}\right)$ \\
$\Xi^{0}(u s s)$ & $a\left(\phi_{u u}^{2}+2 \phi_{s u}^{2}+2 \varphi_{s u}^{2}\right)$ & $a\left(\phi_{u d}^{2}+\varphi_{u d}^{2}+2 \phi_{s d}^{2}+2 \varphi_{s d}^{2}\right)$ & $a\left(\phi_{u s}^{2}+\varphi_{u s}^{2}+2 \phi_{s s}^{2}\right)$ \\
\hline
\end{tabular}

TABLE I. The sea quark distribution functions for the octet baryons. The expressions for other octet baryons can be obtained through isospin symmetry.

There are no simple or straightforward rules which could allow incorporation of $x$-dependence in $\chi \mathrm{CQM}$. To this end, instead of using an ab initio approach, we have phenomenologically incorporated the $x$-dependence getting clues from Eichten et al. [10, Isgur [3] et al. and Le Yaouanc et al. 44. The $x$-dependent sea quark distribution functions can be now expressed as $\bar{u}^{B}(x)=\bar{u}^{B}(1-x)^{10}, \bar{d}^{B}(x)=d^{B}(1-x)^{7}, \bar{s}^{B}(x)=s^{B}(1-x)^{8}$ which together with the valence quark distribution functions give the flavor structure of the baryon as

$$
q^{B}(x)=q_{\text {val }}^{B}(x)+\bar{q}^{B}(x),
$$

where $q=u, d, s$. Using the sea quark distribution functions from Table I, the quark sea asymmetries $\bar{u}(x)-\bar{d}(x)$ and $\bar{d}(x) / \bar{u}(x)$ can also be calculated at different $x$ values. We have already discussed the inclusion of $x$-dependence in detail and compared our results with the experimental data for the case of nucleon in Ref. [27. In the present communication however, we have extended our calculations to the case of other octet baryons for which experimental data is not yet available.

The $x$-dependence of the structure functions $F_{1}$ and $F_{2}$ can be calculated from

$$
\begin{aligned}
& F_{2}^{B}(x)=x \sum_{u, d, s} e_{q}^{2}\left[q^{B}(x)+\bar{q}^{B}(x)\right], \\
& F_{1}^{B}(x)=\frac{1}{2 x} F_{2}^{B}(x),
\end{aligned}
$$


where $e_{q}$ is the charge of the quark $q\left(e_{u}=\frac{2}{3}\right.$ and $\left.e_{d}=e_{s}=-\frac{1}{3}\right)$. In terms of the quark distribution functions, the structure function $F_{2}$ for any baryon can be expressed as

$$
F_{2}^{B}(x)=\frac{4}{9} x\left(u^{B}(x)+\bar{u}^{B}(x)\right)+\frac{1}{9} x\left(d^{B}(x)+\bar{d}^{B}(x)+s^{B}(x)+\bar{s}^{B}(x)\right) .
$$

Several important quantities can be obtained from the structure functions of different isospin multiplets. For example, for the case of proton and neutron we have

$$
\begin{aligned}
\frac{F_{2}^{p}(x)-F_{2}^{n}(x)}{x} & =\frac{4}{9}\left(u_{\mathrm{val}}^{p}(x)-u_{\mathrm{val}}^{n}(x)+2 \bar{u}^{p}(x)-2 \bar{u}^{n}(x)\right)+\frac{1}{9}\left(d_{\mathrm{val}}^{p}(x)+s_{\mathrm{val}}^{p}(x)\right. \\
& \left.-d_{\mathrm{val}}^{n}(x)-s_{\mathrm{val}}^{n}(x)+2 \bar{d}^{p}(x)+2 \bar{s}^{p}(x)-2 \bar{d}^{n}(x)-2 \bar{s}^{n}(x)\right) .
\end{aligned}
$$

The Gottfried integral $I_{G}^{p n}$ [28] can be expressed in terms of the sea quarks as follows

$$
I_{G}^{p n}=\int_{0}^{1} \frac{F_{2}^{p}(x)-F_{2}^{n}(x)}{x} d x=\frac{1}{3}+\frac{2}{3} \int_{0}^{1}\left[\bar{u}^{p}(x)-\bar{d}^{p}(x)\right] d x,
$$

where we have used the following normalization conditions

$$
\begin{gathered}
\int_{0}^{1} u_{\mathrm{val}}^{p}(x) d x=2, \quad \int_{0}^{1} d_{\mathrm{val}}^{p}(x) d x=1, \int_{0}^{1} s_{\mathrm{val}}^{p}(x) d x=0, \\
\int_{0}^{1} u_{\mathrm{val}}^{n}(x) d x=1, \int_{0}^{1} d_{\mathrm{val}}^{n}(x) d x=2, \int_{0}^{1} s_{\mathrm{val}}^{n}(x) d x=0, \\
\int_{0}^{1} \bar{d}^{n}(x) d x=\int_{0}^{1} \bar{u}^{p}(x) d x, \quad \int_{0}^{1} \bar{u}^{n}(x) d x=\int_{0}^{1} \bar{d}^{p}(x) d x, \quad \int_{0}^{1} \bar{s}^{n}(x) d x=\int_{0}^{1} \bar{s}^{p}(x) d x .
\end{gathered}
$$

Similarly, for the case of other octet baryons the following normalization conditions

$$
\begin{array}{ccc}
\int_{0}^{1} u_{\text {val }}^{\Sigma^{+}}(x) d x=2, & \int_{0}^{1} d_{\text {val }}^{\Sigma^{+}}(x) d x=0, & \int_{0}^{1} s_{\text {val }}^{\Sigma^{+}} d x=1 \\
\int_{0}^{1} u_{\text {val }}^{\Sigma^{0}}(x) d x=1, & \int_{0}^{1} d_{\text {val }}^{\Sigma^{0}}(x) d x=1, & \int_{0}^{1} s_{\text {val }}^{\Sigma^{0}}(x) d x=1 \\
\int_{0}^{1} u_{\text {val }}^{\Xi^{0}}(x) d x=1, & \int_{0}^{1} d_{\text {val }}^{\Xi^{0}}(x) d x=0, & \int_{0}^{1} s_{\text {val }}^{\Xi^{0}}(x) d x=2
\end{array}
$$

lead to the other Gottfried integrals in terms of the sea quarks

$$
\begin{aligned}
I_{G}^{\Sigma^{+} \Sigma^{0}} & \equiv \int_{0}^{1} \frac{F_{2}^{\Sigma^{+}}(x)-F_{2}^{\Sigma^{0}}(x)}{x} d x=\frac{1}{3}+\frac{2}{9} \int_{0}^{1}\left[4 \bar{u}^{\Sigma^{+}}(x)+\bar{d}^{\Sigma^{+}}(x)-4 \bar{u}^{\Sigma^{0}}(x)-\bar{d}^{\Sigma^{0}}(x)\right] d x, \\
I_{G}^{\Sigma^{0} \Sigma^{-}} & \equiv \int_{0}^{1} \frac{F_{2}^{\Sigma^{0}}(x)-F_{2}^{\Sigma^{-}}(x)}{x} d x=\frac{1}{3}+\frac{2}{9} \int_{0}^{1}\left[4 \bar{u}^{\Sigma^{0}}(x)+\bar{d}^{\Sigma^{0}}(x)-4 \bar{d}^{\Sigma^{+}}(x)-\bar{u}^{\Sigma^{+}}(x)\right] d x, \\
I_{G}^{\Xi^{0} \Xi^{-}} & \equiv \int_{0}^{1} \frac{F_{2}^{\Xi^{0}}(x)-F_{2}^{\Xi^{-}}(x)}{x} d x=\frac{1}{3}+\frac{2}{3} \int_{0}^{1}\left[\bar{u}^{\Xi^{0}}(x)-\bar{d}^{\Xi^{0}}(x)\right] d x .
\end{aligned}
$$

It is clear from Eqs. 11 and $\left(14\right.$, the flavor symmetric sea leads to the Gottfried sum rule $I_{G}=\frac{1}{3}$ with $\bar{u}^{B}=\bar{d}^{B}$.

After having detailed the contribution of the quark sea and the various asymmetries in the octet baryons of different quark structure, we now discuss the variation of these quantities with the Bjorken variable $x$. For the numerical calculation of the sea quark distribution functions of the octet baryons, we have used the same set of input parameters as detailed in our earlier calculations [13, 16, 27, 29. In Fig. 1. we have shown the variation of the sea quark distributions $x \bar{u}(x), x \bar{d}(x)$ and $x \bar{s}(x)$ with the Bjorken scaling variable $x$ for $p(u u d), \Sigma^{+}(u u s), \Sigma^{0}(u d s)$ and $\Xi^{0}($ uss $)$. From a cursory look at the plots, one can easily find out that

$$
\bar{d}^{p}(x)>\bar{u}^{p}(x)>\bar{s}^{p}(x)
$$




$$
\begin{gathered}
\bar{d}^{\Sigma^{+}}(x)>\bar{u}^{\Sigma^{+}}(x) \approx \bar{s}^{\Sigma^{+}}(x), \\
\bar{d}^{\Sigma^{0}}(x)>\bar{u}^{\Sigma^{0}}(x)>\bar{s}^{\Sigma^{0}}(x), \\
\bar{d}^{\Xi^{0}}(x)>\bar{u}^{\Xi^{0}}(x)>\bar{s}^{\Xi^{0}}(x),
\end{gathered}
$$

showing a clear quark sea asymmetry as observed in the DIS experiments [6, 17, 18. These distributions clearly indicate that our results pertaining to the quark sea asymmetry seem to be well in line with the expected results.

A careful study of the plots brings out several interesting points. As already mentioned in the introduction, the sea quarks do not contribute at higher values of $x$, therefore in Fig. 1, we have taken the region $x=0-0.5$. Beyond this $x$ region the contribution of the sea quarks is negligible and the contributions should be completely dominated by the valence quarks. The difference between the various sea distributions is observed to be maximum at $x \approx 0.1$. As the value of $x$ increases, the difference between the sea contributions decreases in all the cases which is in line with the observations of other models [20, 22, 24].

The general aspects of the variation of the magnitudes of the sea quark distribution functions $\bar{u}(x), \bar{d}(x)$ and $\bar{s}(x)$ for the octet baryons are able to explain some of the well known experimentally measurable quantities, for example, $\bar{d}^{B}(x)-\bar{u}^{B}(x), \bar{d}^{B}(x) / \bar{u}^{B}(x)$ and the Gottfried integral. These quantities not only provide important constraint on a model that attempts to describe the origin of the quark sea but also provide a direct determination of the presence of significant amount of quark sea in the low $x$ region. In Fig. 2. the $\chi$ CQM results for the $\bar{d}^{B}(x)-\bar{u}^{B}(x)$ and the Gottfried integrals have been plotted at different $x$ values. It is clear from the plots that when $x$ is small $\bar{d}^{B}(x)-\bar{u}^{B}(x)$ asymmetries are large implying the dominance of sea quarks in the low $x$ region. In fact, the sea quarks dominate only in the region where $x$ is smaller than 0.3 . At the values $x>0.3, \bar{d}-\bar{u}$ tends to 0 implying that there are no sea quarks in this region. The contribution of the quark sea in the case of $\Sigma^{0}$ is particularly interesting because of its flavor structure which has equal numbers of $u, d$ and $s$ quarks in its valence structure. Unlike the other octet baryons, where the $\bar{d}(x)-\bar{u}(x)$ asymmetry decreases continuously with the $x$ values, the asymmetry in this case first increases and then for values of $x>0.1$ it decreases. However, it is interesting to observe the the asymmetry peak in this case which matches with our other predictions where the contribution of the quark sea is maximum at $x \approx 0.1$

A measurement of the Gottfried integral [6, 17, for the case of nucleon has shown a clear violation of Gottfried sum rule from $\frac{1}{3}$ which can find its explanation in a global quark sea asymmetry $\int_{0}^{1}(\bar{d}(x)-\bar{u}(x)) d x$. Similarly, for the case of $\Sigma^{+}, \Sigma^{0}$, and $\Xi^{0}$, the Gottfried sum rules should read $I_{G}^{\Sigma^{+}} \Sigma^{0}=\frac{1}{3}, I_{G}^{\Sigma^{0}} \Sigma^{-}=\frac{1}{3}$ and $I_{G}^{\Xi^{0}} \Xi^{-}=\frac{1}{3}$ if the quark sea was symmetric. However, due to the $\bar{d}(x)-\bar{u}(x)$ asymmetry in the case of octet baryons, a lower value of the Gottfried integrals is obtained. We have plotted the results in Fig. 2. In the case of nucleon the results are in good agreement with the experimental data [17] as already presented in [27. The quality of numerical agreement in the other cases can be assessed only after the data gets refined. Further, this phenomenological analysis strongly suggests an important role for the quark sea at low value of $x$. New experiments aimed at measuring the flavor content of the other octet baryons are needed for profound understanding of the nonperturbative properties of QCD.

To summarize, in order to investigate the effects of "quark sea", we have calculated the sea quark distribution functions for the octet baryons in the chiral constituent quark model $(\chi \mathrm{CQM})$. The Bjorken scaling variable $x$ has been incorporated phenomenologically to enlarge the scope of model and to understand the range of $x$ where quark sea effects are important. Implications of the quark sea have also been studied to estimate the quark sea asymmetries like $\bar{d}(x)-\bar{u}(x), \bar{d}(x) / \bar{u}(x)$ and Gottfried integral. The results justify our conclusion regarding the importance of quark sea at small values of $x$.

In conclusion, the results obtained for the quark distribution functions reinforce our conclusion that $\chi \mathrm{CQM}$ is able to generate qualitatively as well as quantitatively the requisite amount of quark sea. This can perhaps be substantiated by a measurement of the quark distribution functions of the other octet baryons.

\section{ACKNOWLEDGMENTS}

H.D. would like to thank Department of Science and Technology, Government of India, for financial support.

[1] E.D. Bloom et al., Phys. Rev. Lett. 23, 930 (1969); M. Breidenbach et al., Phys. Rev. Lett. 23, 935 (1969).

[2] A. De Rujula, H. Georgi and S.L. Glashow, Phys. Rev. D 12, 147 (1975).

[3] N. Isgur, G. Karl and R. Koniuk, Phys. Rev. Lett. 41, 1269 (1978);N. Isgur and G. Karl, Phys. Rev. D 21, 3175 (1980); P.Geiger and N. Isgur, Phys. Rev. D 55, 299 (1997); N. Isgur, Phys. Rev. D 59, 034013 (1999).

[4] A. Le Yaouanc, L. Oliver, O. Pene and J.C. Raynal, Phys. Rev. D 12, 2137 (1975); 15, 844 (1977). 

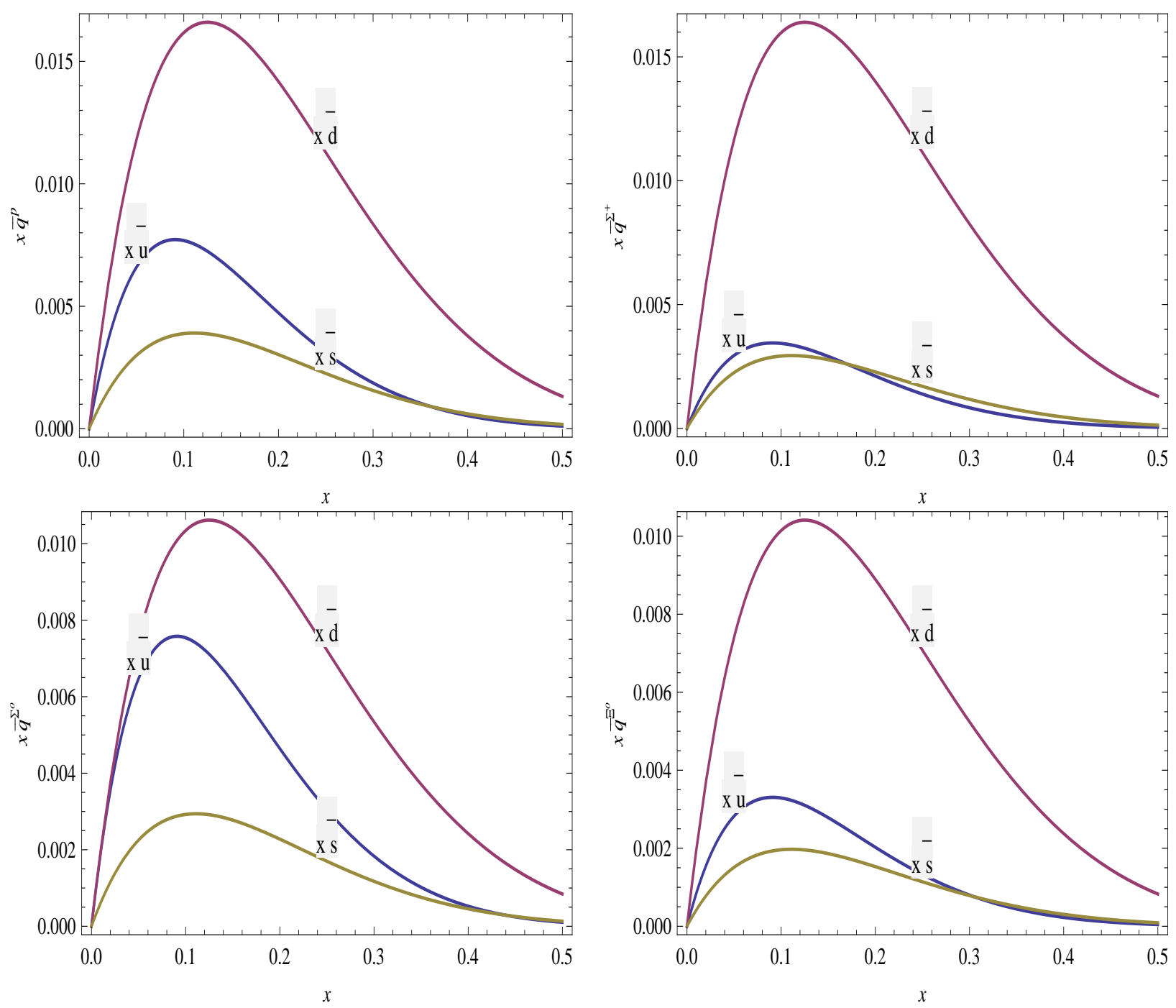

FIG. 1. Sea quark distribution functions as a function of Bjorken scaling variable $x$ for the $p, \Sigma^{+}, \Sigma^{0}$, and $\Xi^{0}$, respectively.

[5] EMC Collaboration, J. Ashman et al., Phys. Lett. B 206, 364 (1988); Nucl. Phys. B 328, 1 (1989); SMC Collaboration, B. Adeva et al., Phys. Lett. B 302, 533 (1993); P. Adams et al., Phys. Rev. D 56, 5330 (1997); E142 Collaboration, P.L. Anthony et al., Phys. Rev. Lett. 71, 959 (1993); E143 Collaboration, K. Abe et al., Phys. Rev. Lett. 75, 391 (1995).

[6] New Muon Collaboration, P. Amaudruz et al., Phys. Rev. Lett. 66, 2712 (1991); M. Arneodo et al., Phys. Rev. D 50, R1 (1994).

[7] NA51 Collaboration, A. Baldit et al., Phys. Lett. B 253, 252 (1994).

[8] J.D. Sullivan, Phys. Rev. D 5, 1732 (1972); A.W. Thomas, Phys. Lett. B 126, 97 (1983); J. Magnin and H.R. Christiansen, Phys. Rev. D 61, 054006 (2000).

[9] S. Weinberg, Physica A 96, 327 (1979); A. Manohar and H. Georgi, Nucl. Phys. B 234, 189 (1984).

[10] E.J. Eichten, I. Hinchliffe and C. Quigg, Phys. Rev. D 45, 2269 (1992).

[11] T.P. Cheng and Ling Fong Li, Phys. Rev. Lett. 74, 2872 (1995); T.P. Cheng and Ling Fong Li, Phys. Rev. D 57, 344 (1998).

[12] X. Song, J.S. McCarthy, and H.J. Weber, Phys. Rev. D 55, 2624 (1997); X. Song, Phys. Rev. D 57, 4114 (1998).

[13] H. Dahiya and M. Gupta, Phys. Rev. D 64, 014013 (2001); 67, 074001 (2003); Int. Jol. of Mod. Phys. A, 1929, 5027 (2004); H. Dahiya, M. Gupta, and J.M.S. Rana, Int. Jol. of Mod. Phys. A, 21, 214255 (2006); H. Dahiya and M. Gupta, Phys. Rev. D 78, 014001 (2008).

[14] H. Dahiya and M. Gupta, Phys. Rev. D 66, 051501(R) (2002); 67, 114015 (2003).

[15] J. Linde, T. Ohlsson, and H. Snellman, Phys. Rev. D 57, 452 (1998); 57, 5916 (1998).

[16] N. Sharma, H. Dahiya, P.K. Chatley, and M. Gupta, Phys. Rev. D 79, 077503 (2009); N. Sharma, H. Dahiya, and P.K. Chatley Eur. Phys. J. A 44, 125 (2010); N. Sharma, H. Dahiya, P.K. Chatley, and M. Gupta Phys. Rev. D 81, 073001 (2010). 

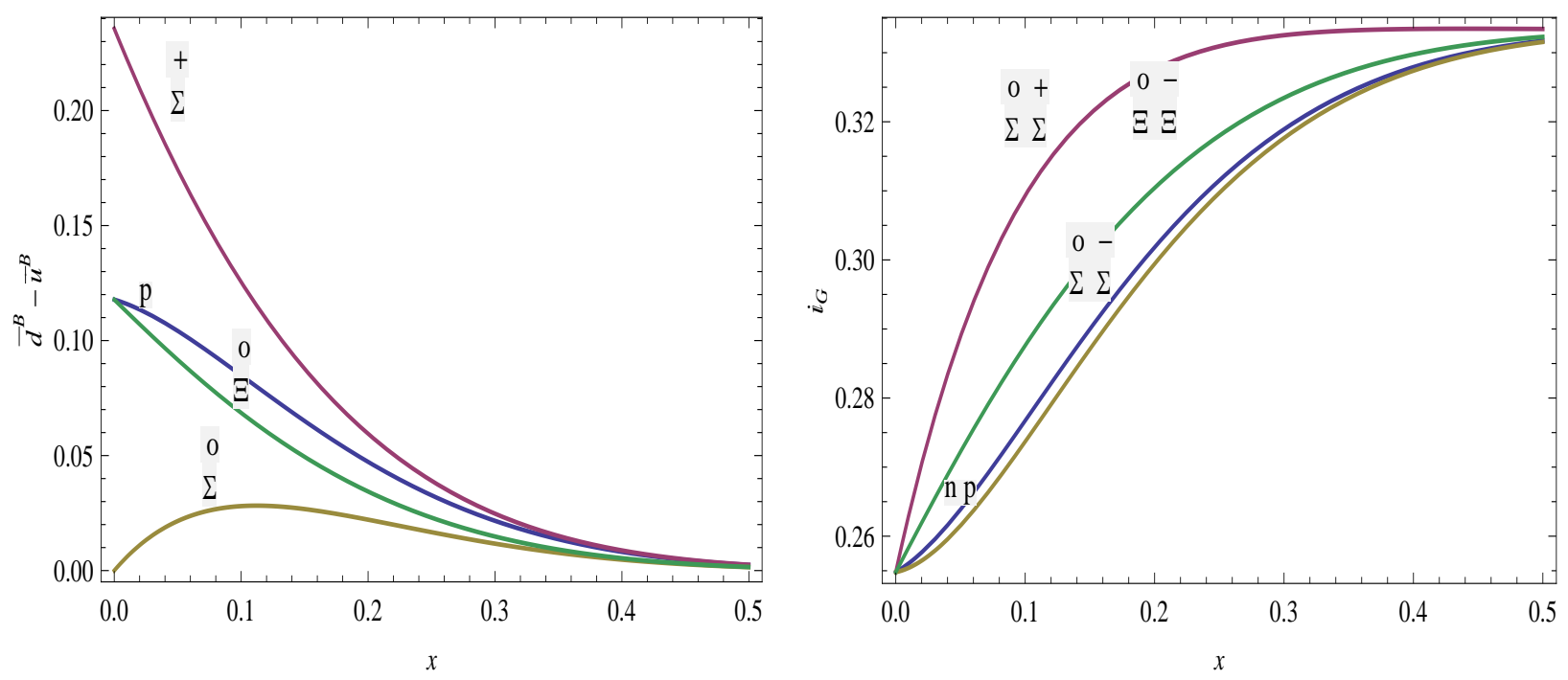

FIG. 2. Sea quark asymmetry and Gottfried integrals as a function of Bjorken scaling variable $x$ for the octet baryons.

[17] E866/NuSea Collaboration, E.A. Hawker et al., Phys. Rev. Lett. 80, 3715 (1998); J.C. Peng et al., Phys. Rev. D 58, 092004 (1998); R. S. Towell et al., Phys. Rev. D 64, 052002 (2001).

[18] HERMES Collaboration, K. Ackerstaff et al., Phys. Rev. Lett. 81, 5519 (1998).

[19] M. Alberg, E.M. Henley, and G.A. Miller, Phys.Lett. B 471, 396 (2000); S. Kumano and M. Miyama, Phys. Rev. D 65, 034012 (2002); Fu-Guang Cao and A. I. Signal, Phys. Rev. D 68, 074002 (2003); F. Huang, R.-G. Xu, B.-Q. Ma, Phys. Lett. B 602, 67 (2004); B. Pasquini, S. Boffi, Nucl. Phys. A 782, 86 (2007).

[20] M. Wakamatsu, Phys. Rev. D 44, R2631 (1991); M. Wakamatsu, Phys. Rev. D 46, 3762 (1992); H. Weigel, Phys. Rev. D 55, 6910 (1997); M. Wakamatsu and T. Kubota, Phys. Rev. D 57, 5755 (1998); M. Wakamatsu, Phys. Rev. D 67, 034005 (2003).

[21] Yong Ding, Rong-Guang Xu, and Bo-Qiang Ma, Phys. Rev. D 71, 094014 (2005); Lijing Shao, Yong-Jun Zhang, and Bo-Qiang Ma, Phys. Lett. B 686, 136 (2010).

[22] L.A. Trevisan, C. Mirez, T. Frederico, and L. Tomio, Eur. Phys. J. C 56, 221 (2008); Yunhua Zhang, Lijing Shao, and, Bo-Qiang Ma, Phys. Lett. B 671, 30 (2009); Yunhua Zhang, Lijing Shao, and Bo-Qiang Ma, Nucl. Phys. A 828, 390 (2009).

[23] A.I. Signal and A.W. Thomas, Phys. Rev. D 40, 2832 (1989).

[24] J. Alwall and G. Ingelman, Phys. Rev. D 71, 094015 (2005).

[25] M. Glück, E. Reya, and A. Vogt, Z. Phys. C 67, 433 (1995); M. Glück, E. Reya, M.Stratmann, and W. Vogelsang, Phys. Rev. D 53, 4775 (1996); D. de Florian, C.A. Garcia Canal, and R. Sassot, Nucl. Phys. B 470, 195 (1996).

[26] CDHS Collaboration, H. Abramowicz et al., Z. Phys. C 17, 283(1983); Costa et al., Nucl. Phys. B 297, 244 (1988).

[27] H. Dahiya and M. Gupta, Eur. Phys. J. C 52, 571 (2007).

[28] K. Gottfried, Phys. Rev. Lett. 18, 1174 (1967).

[29] C. Amsler et al., Phys. Lett. B 667, 1 (2008). 